\title{
Complementary therapies for bladder pain syndrome: a systematic review
}

\author{
Tina S. Verghese ${ }^{1,5} \cdot$ Richael Ni Riordain $^{2} \cdot$ Rita Champaneria $^{3} \cdot$ Pallavi M. Latthe $^{4}$
}

Received: 12 July 2015 / Accepted: 26 October 2015 / Published online: 7 December 2015

(C) The Author(s) 2015. This article is published with open access at Springerlink.com

\begin{abstract}
Introduction and hypothesis Bladder pain syndrome is a difficult condition to treat. The purpose of this systematic review is to assess the effectiveness of various complementary therapies available for treatment.

Methods This review was conducted in adherence with Preferred Reporting Items for Systematic Reviews. Citations were retrieved using a comprehensive database search (from inception to July 2014: CINAHL, Cochrane, EMBASE, Medline and SIGEL and grey literature). Studies that fulfilled the inclusion criteria were selected. Eligibility consisted of women with bladder pain syndrome, an intervention of alternative/complementary therapies and an outcome of improvement of symptoms. Information regarding study characteristics and primary outcomes was collated. The Cochrane risk of bias scale was used to evaluate the quality of the studies included.

Results A total of 1,454 citations were identified, 11 studies fulfilled the inclusion criteria (4 randomised control trials [RCTs] and 7 prospective studies). The key interventions
\end{abstract}

Tina S. Verghese

t.s.verghese@bham.ac.uk

1 University of Birmingham, Birmingham, UK

2 Newham University Hospital, Barts Health NHS Trust, Birmingham, UK

3 Birmingham Clinical Trials Unit, Birmingham University, Birmingham, UK

4 Birmingham Women's NHS Foundation Trust, Birmingham, UK

5 School of Clinical and Experimental Medicine, College of Medical and Dental Sciences, The University of Birmingham, B15

2TT Birmingham, UK studied were acupuncture, relaxation therapy, physical therapy, hydrogen-rich therapy, diet and nitric oxide synthetase. Conclusion Therapies with the potential for benefit in patients with bladder pain syndrome are dietary management, acupuncture and physical therapy. These findings were obtained from small studies and hence caution is advised. Robustly designed multicentre RCTs on these complementary therapies are needed to guide patients and clinicians.

Keywords Alternative or complementary therapies . Myofascial physical therapies $\cdot$ Acupuncture $\cdot$ Bladder pain syndrome

\section{Introduction}

The European Society for the Study of Interstitial Cystitis/ Bladder Pain Syndrome in 2008 defined bladder pain syndrome (BPS) as pelvic pain, pressure or discomfort perceived to be related to the bladder, lasting for at least 6 months, and accompanied by at least one other urinary symptom [1]. Urinary symptoms include the persistent urge to void or frequency, in the absence of other identifiable causes. The International Urogynaecological Association (IUGA) and the International Continence Society (ICS) produced a joint report on terminologies by Haylen et al. in 2010, defining bladder pain as a "complaint of supra pubic or retro-pubic pressure, discomfort or pain, associated with the bladder, generally aggravated by bladder filling. The symptom may persist or alleviate after voiding." [2]. An estimated 400,000 people in the UK suffer from BPS, the majority being women [3]. There is no definitive evidence to support an autoimmune, inflammatory, structural or infectious aetiology. Consequently, treating these patients is often challenging. 
The interest in complementary and alternative therapies among patients is high and the number of effective treatments available for BPS are few [4]. Complementary and alternative therapies are an essential addition to the therapeutic armamentarium and include dietary modification, bladder training, acupuncture and stress reduction [5]. In 1995, complementary and alternative medicine (CAM) was defined by the National Institutes of Health (NIH) Center for Complementary and Alternative Medicine as, those treatments and healthcare modalities not widely taught or practised in medical schools or hospitals, and not usually reimbursed by medical insurance companies [6].

Our aim was to conduct a systematic review to evaluate the effectiveness of complementary therapies in the treatment of BPS.

\section{Materials and methods}

This review was planned, conducted and reported in adherence with widely recommended methods [7]. No ethical approval was needed.

\section{Identification of studies}

The following bibliographic databases were searched, from database inception to July 2014: CINAHL, Cochrane, EMBASE, Medline and SIGEL. Search strategies consisted of MeSH subheadings, text words and word variations for the concepts of BPS and alternative/complementary therapies. The basic search strategy was adapted to suit the database being searched. Librarians at the Royal College of Obstetricians and Gynaecologists (RCOG) performed the database searches. The search terms utilised by the RCOG: (Interstitial cystitis OR painful bladder syndrome OR bladder pain syndrome) AND Analgesia OR Pain relief OR Diet OR caffeine OR citrus OR Alcohol OR Smoking OR nicotine OR tobacco OR Physical therapy OR massage OR cupping OR exercise OR bladder training OR pelvic floor exercise OR Kegel exercise OR Stress management OR Psychology OR CBT OR talking OR counselling OR forums OR support groups OR acupuncture OR L-arginine. The search was restricted to "humans" and "females". Bibliographies of relevant primary articles were also searched to identify any articles missed by the electronic searches. The conference proceedings and abstracts of the International Continence Society (2004-2014) and International Urogynecological Association meetings (2004-2014) were also searched. A comprehensive database was constructed using Endnote X7.2 for Windows, released 30 September 2014; Mac release date: 30 September 2014 [8] to store all references identified. No language restrictions were applied.

\section{Study selection and data extraction}

Studies were selected following a two-step process. Firstly, the citations identified by the electronic bibliographic database searches were screened, based on their titles and abstracts. Full text papers of eligible abstracts were retrieved. Once full text papers had been located, we determined whether they fulfilled our predetermined inclusion criteria:

Population: women with BPS or interstitial cystitis (IC). Intervention: complementary or alternative therapies Comparator: no treatment, another therapy Outcome: improvement in bladder symptoms Study designs: randomised controlled trial (RCT), cohort studies, case control studies, case series Exclusion criteria: pharmacological therapy, intravesical therapy, hydrodistention, tibial nerve stimulation

Two reviewers (TV, RNR) independently assessed the full text papers to determine if they met the above criteria. Any disagreements regarding the eligibility of a paper were solved through either consensus or arbitration by a third reviewer (PML). Data from the manuscripts included were extracted independently onto a pre-designed pro-forma. The literature search was thorough and without language restrictions. The grey literature was also searched and the data were extracted by two reviewers independently to reduce bias.

Data were collated on study characteristics, including methods of recruitment, patient characteristics, details of complementary therapy, outcomes and results. We contacted primary authors via email for any further information/ clarification that was needed.

\section{Methodological quality}

The methodological quality of all the papers fulfilling the inclusion criteria was assessed. Quality was defined as the confidence that the study design, conduct and analysis minimised bias in the estimation of effectiveness. Quality was assessed using existing checklists and scales $[9,10]$.

The methodological quality of the RCTs included was assessed using the Cochrane risk bias score [9]. The methodological quality of the non-RCT studies included was assessed using the Newcastle-Ottawa scale [10], where a nonrandomised study was considered to be of high quality if it provided information on selection, comparability, exposure and outcome of the study participants. The quality checklist awards one star as a maximum for all items except comparability, where a maximum of two stars can be awarded. The scale was used to give a quantitative appraisal of overall quality of the non-randomised studies. The score ranged from 0 to 9, with a score of either 0 or 1 for each item. 


\section{Data synthesis}

We grouped studies according to their type of intervention and the comparison. The comparisons were with no intervention, with placebo or with another intervention. The outcome measures were based on the responses from questionnaires such as the Visual Analogue Score (VAS) [11], the Interstitial Cystitis Symptom Index (ICSI)/O'Sant Leary questionnaire [12], the Interstitial Cystitis Problem Index (IPSI) [12] and the maximum voided volume (MVV) on the frequency volume chart.

\section{Results}

Out of the 1,454 citations identified by electronic literature searches, 11 studies that fulfilled the inclusion criteria were included in the systematic review (Fig. 1); 4 were randomized controlled trials (RCTs) and 7 were prospective studies. Table 1 provides a summary of the characteristics of the studies included. The mean age of the women ranged between 43 and 64 years. The criteria for the diagnosis of BPS varied in the studies included. Some studies diagnosed BPS clinically based on bladder pain scale, frequency and urgency. Other studies confirmed PBS based on the cystoscopic and hydrodistention findings. Because of the varied definitions utilised in last 5 years, we set the inclusion criteria for women with a diagnosis of BPS to be based on one of the following definitions: Hanash and Pool [13, 14], Messing and Stamey [15], the ICS [2] or NIH criteria [16], or the ESSIC definition (Table 1) [1]. Outcomes were documented as per the symptom-specific scores (VAS score, ICSI and ICPI).

Tables 2 and 3 provide details of the methodological quality of the RCTs included. Table 4 summarises details of the quality of the non-randomised studies included.
Fig. 1 Flow process of the review
Total number of citations identified through electronic

searches $(n=1454)$

Citations excluded after screening titles/ abstracts $(n=1425)$

Articles retrieved for detailed evaluation $(n=30)$

Articles excluded: $n=19$

-Incorrect population $\quad n=1$

-Duplicates

$\mathrm{n}=2$

-No useable data $\quad n=10$

-Not locatable $\quad n=6$

Articles included in the systematic review $(n=11)$ 


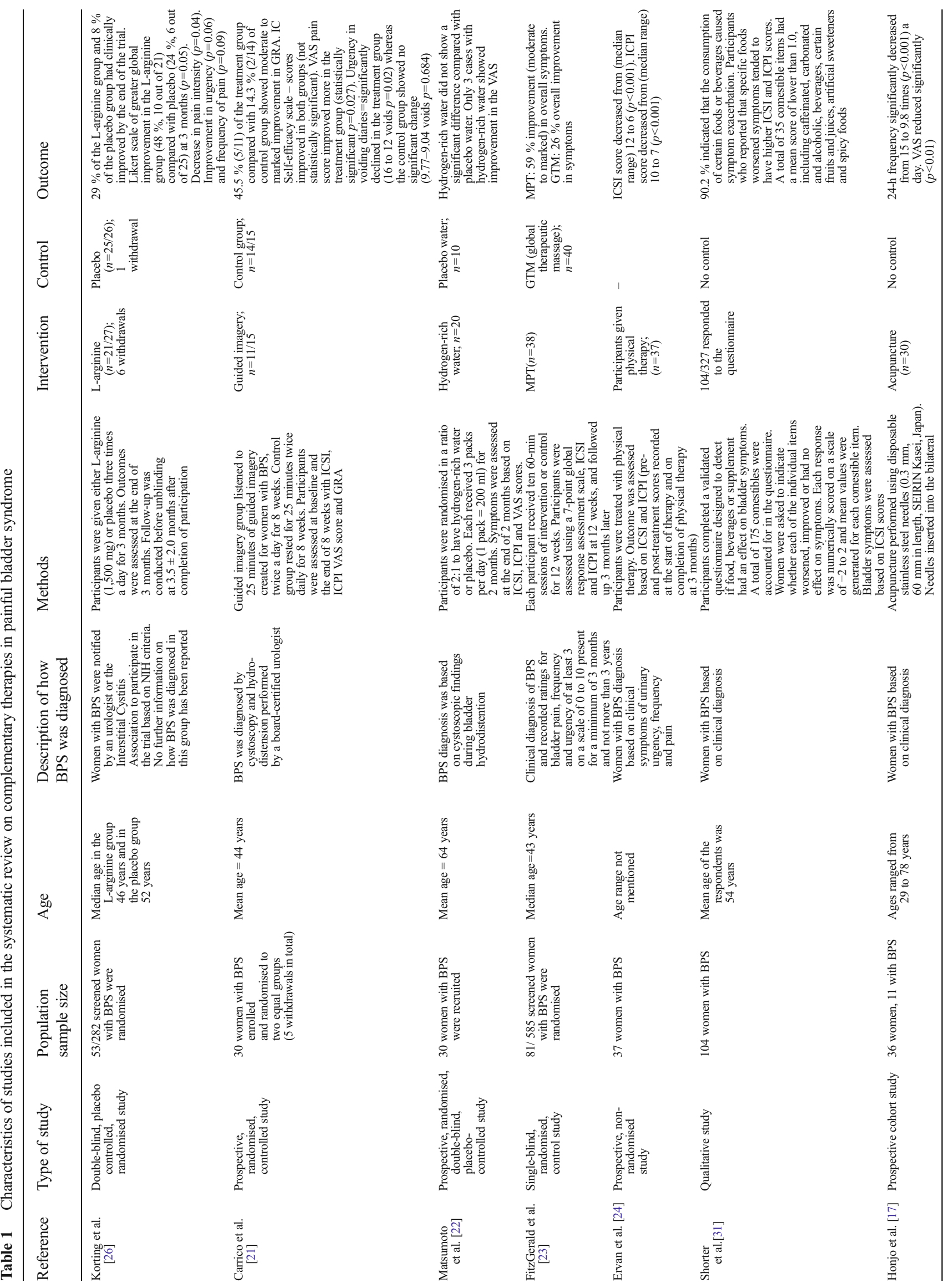




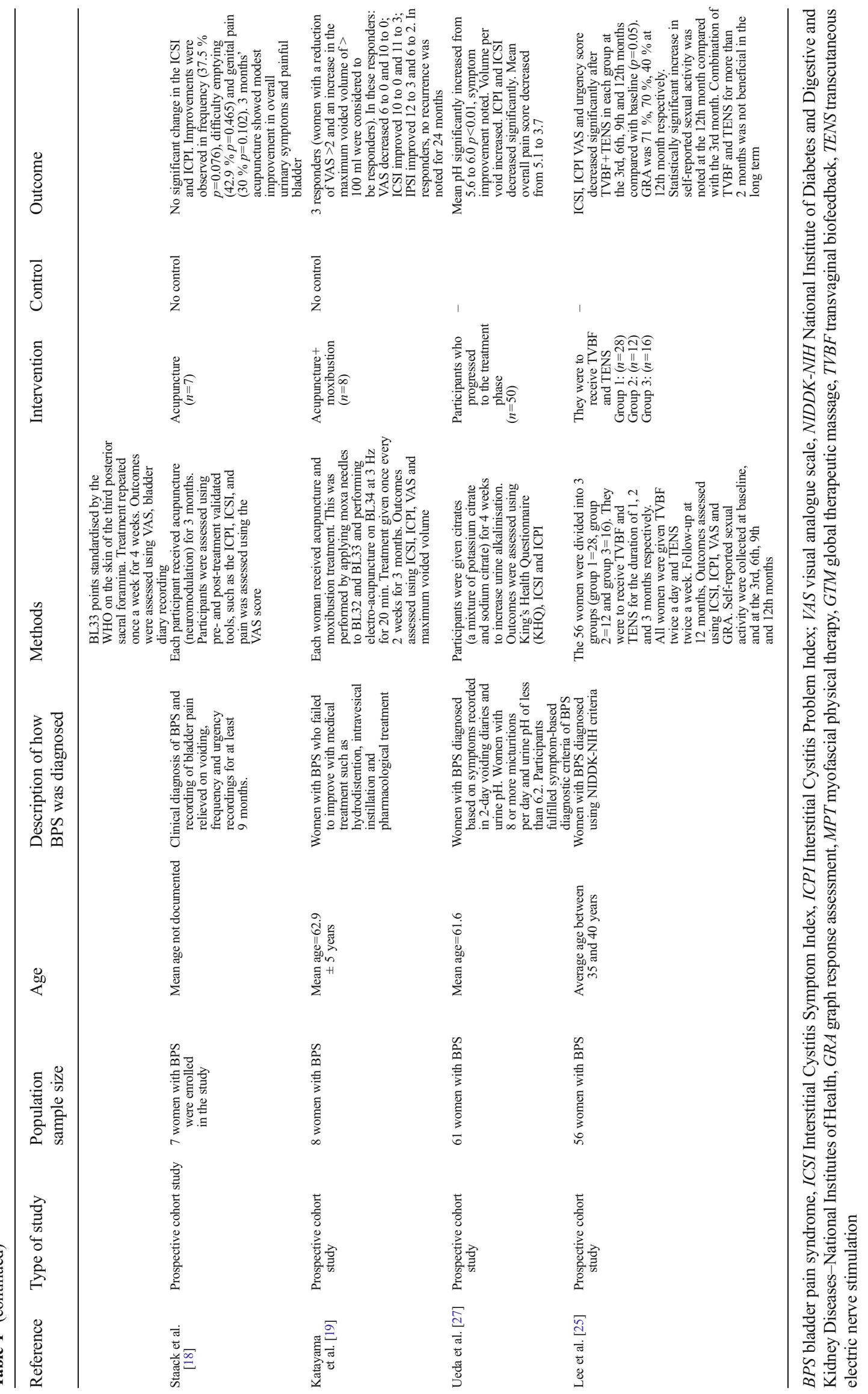


Table 2 Risk of bias summary of the randomised control studies included

KEY:

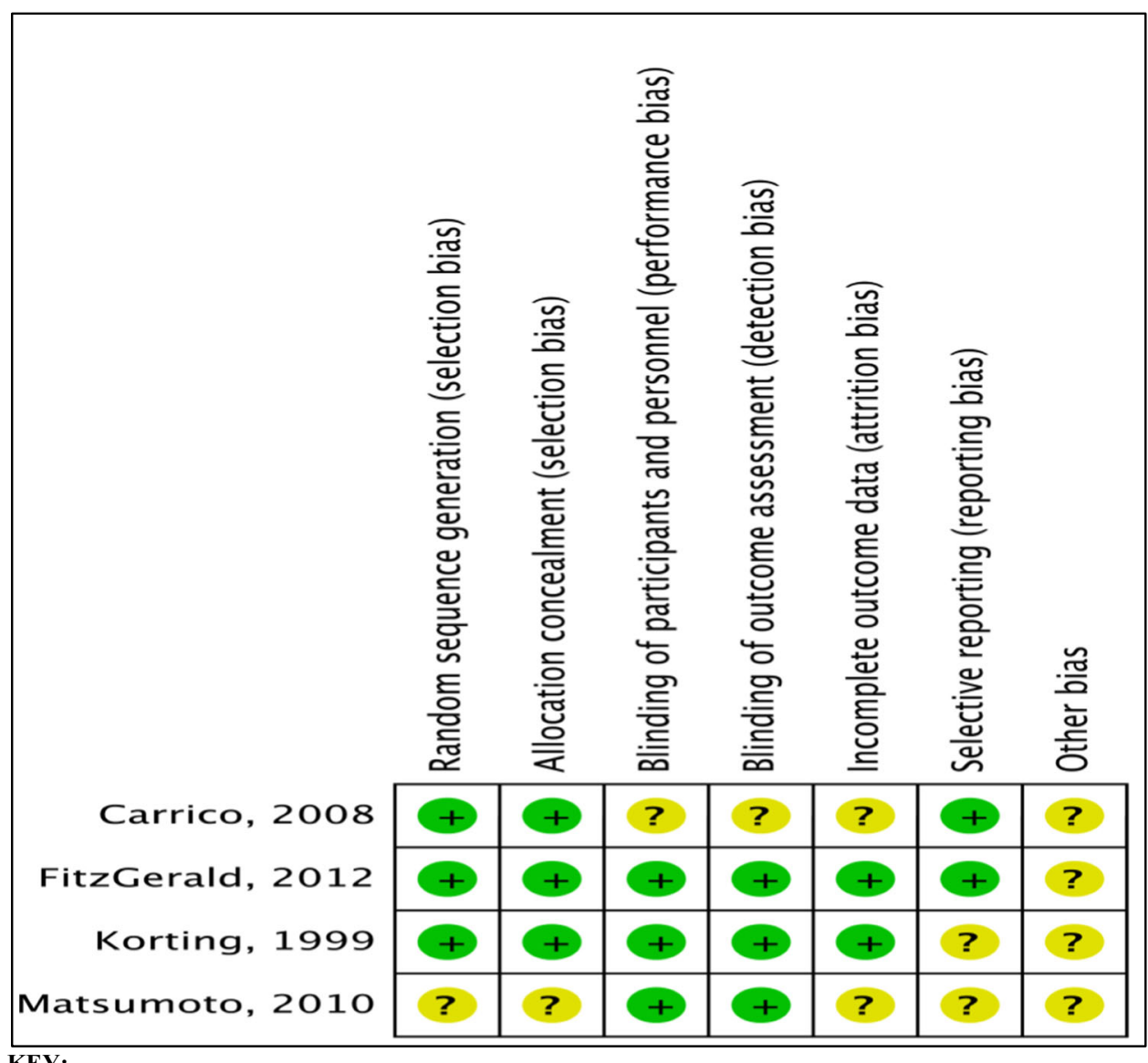

KEY:

+ indicates low risk of bias

- indicates high risk of bias

? unclear risk of bias

+ indicates low risk of bias

- indicates high risk of bias

? unclear risk of bias

\section{Acupuncture and relaxation therapy}

Honjo et al. investigated the clinical impact of sacral acupuncture on urinary sensory dysfunction on a cohort of 36 patients, 11 of whom had BPS (see Table 1). The treatment was repeated weekly for 4 weeks. At the end of treatment there was a significant decrease in the 24-h frequency and VAS for pain $(p<0.001)$ [17]. However, the results for the BPS and overactive bladder patients were presented together, preventing an assessment of symptoms in patients with BPS alone. Similarly, Staack et al. reported on a pilot study of seven IC patients in which 3 months of weekly acupuncture treatment with electric stimulation led to modest improvement in the urinary frequency, voiding difficulty and abdominal/genital pain [18]. Katayama et al. examined the effectiveness of acupuncture and moxibustion in 8 women with refractory BPS.
$38 \%$ of women showed improvement in symptoms after 3 months [19].

In a study conducted at the University of Tennessee, 20 patients with IC were hypnotised every 2 weeks for 2 months. Outcomes measured were a reduction in the analgesic requirements, increase in sleep time and quality of life (QoL). The majority of patients $(95 \% ; 19$ out of 20$)$ were amenable to hypnotherapy and subsequently experienced pain reduction. Five patients reported a significant improvement (8-10 to $0-1$ out of 10$)$ on VAS and $85 \%(n=17$ out of 20$)$ were able to reduce their intake of analgesics by $25-50 \%$ [20]. Carrico et al. conducted a pilot RCT in which more than $45 \%$ of the treatment group responded to "guided imagery", with a moderate or marked improvement in the global response rates (Table 1). In addition, the treatment group had a significant reduction in their ICSI $(p=0.006)$ and ICPI scores $(p=0.004)$ [21]. 
Table 3 Risk of bias graph of the randomized control studies included

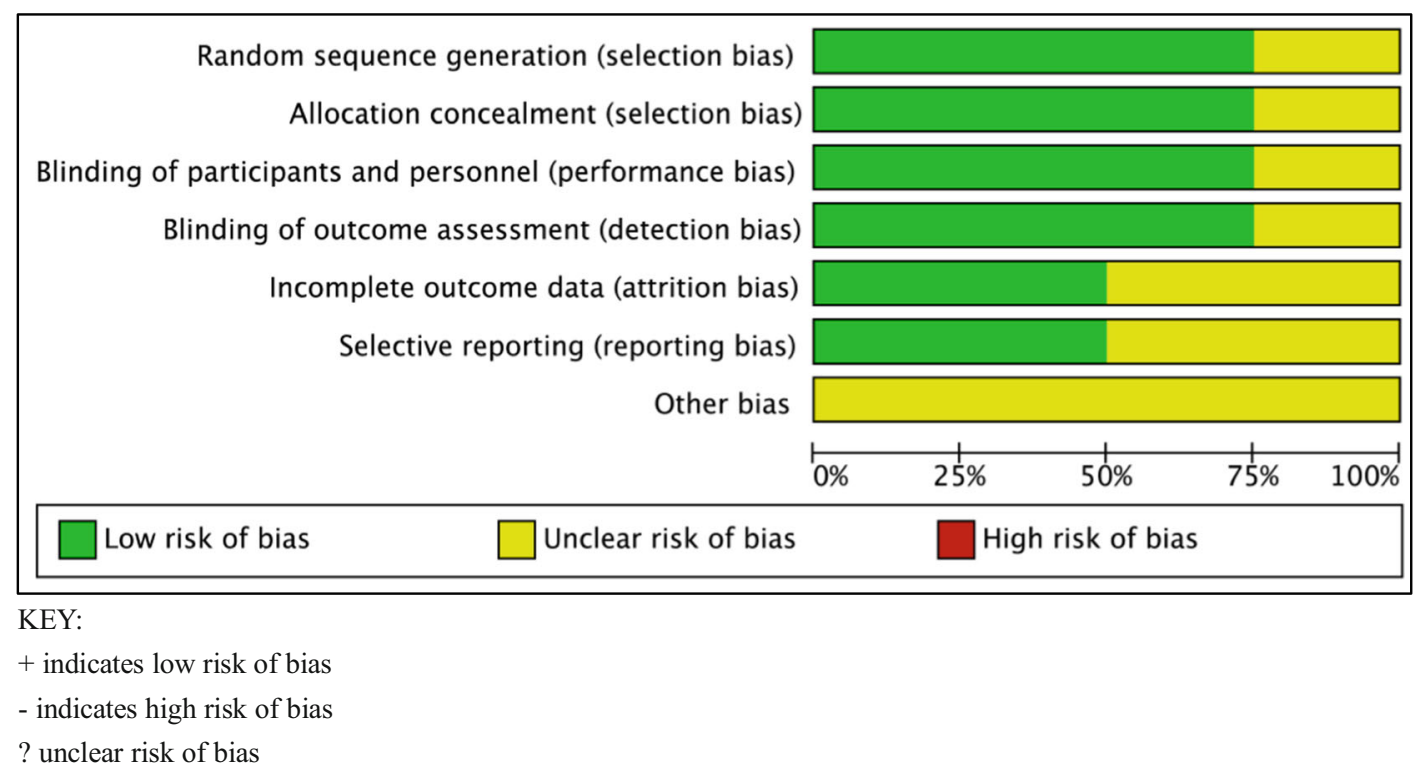

\section{Hydrogen-rich therapy}

Matsumoto et al. conducted an RCT (30 women) of hydrogenrich water versus placebo water for 2 months. The authors describe the process of the production of hydrogen-rich water. This was produced by passage through a reverse osmosis, an ion-exchange resin, and an ultrafiltration membrane. Hydrogen-rich pure water was then produced from dissolving hydrogen gas directly into pure water. To prevent the loss of hydrogen, the water is sealed in aluminium pouches and stored at room temperature. The study demonstrated no significant differences between treatment and placebo (Table 1) [22].

\section{Physical therapy}

FitzGerald et al. conducted a multicentre RCT ( $n=81$ women) to determine the efficacy of myofascial physical therapy (MPT) compared with global therapeutic massage (GTM) [23]. The MPT group underwent connective tissue manipulation to all body wall tissues of the abdominal wall, back, buttocks and thighs that were clinically found to have connective tissue abnormalities to painful myofascial trigger points. Manipulation was applied bilaterally with the patient in the prone and then repeated in the supine position. This was performed until a texture change was noted. Manual trigger point release techniques were utilized to treat any noted trigger points or scars in the anterior or posterior lower quadrants. In the MPT arm, the therapists tailored the therapy to target patient needs.

Patients randomised to the GTM group received weekly massages consisting of full-body Western massage for $1 \mathrm{~h}$. The therapist utilised techniques such as effleurage, petrissage, friction, tapotement, vibration and kneading.
At the end of 12 weeks, the global response rate was $26 \%$ in the GTM group and $59 \%$ in the MPT $(p=0.0012)$ group. Both groups reported improvement in secondary outcomes, including pain, urgency, frequency and QoL [23].

Similarly Ervan et al. conducted a non-randomised study on women with BPS. The physical therapy included connective tissue mobilisation over the trunk, thighs, buttocks and the release of trigger points, the resolution of adverse neural tension along the pudendal nerve, rehabilitation of the pelvic floor and hip girdle musculature. ICSI scores fell from median range of 12 before treatment to 6 post-therapy $(p<0.001)$. The ICPI scores decreased from 10 to 7 after therapy $(p<0.001)$ [24].

Women with BPS tend to have hypertonic pelvic floor muscle dysfunction. In these women, a combination of transvaginal biofeedback and transcutaneous electric nerve stimulation for more than 2 months has not been shown to have a long-term beneficial effect [25].

\section{L-arginine}

Korting et al. undertook an RCT to investigate the efficacy of L-arginine, which is a substrate for nitric oxide, in which there were no differences between the groups according to intention-to-treat analysis (Table 1) [26].

Ueda et al. in their prospective study administered citrates to elevate the urine $\mathrm{pH}$ (Table 1). Urine alkalinisation was shown to be effective at improving symptoms of BPS [27].

Sacral acupuncture is a safe and promising therapeutic alternative, particularly in patients with PBS who have symptoms that do not respond to conventional treatments [17]. Acupuncture inhibits the transmission of pain and normalizes sensory processing within the peripheral and/or central 


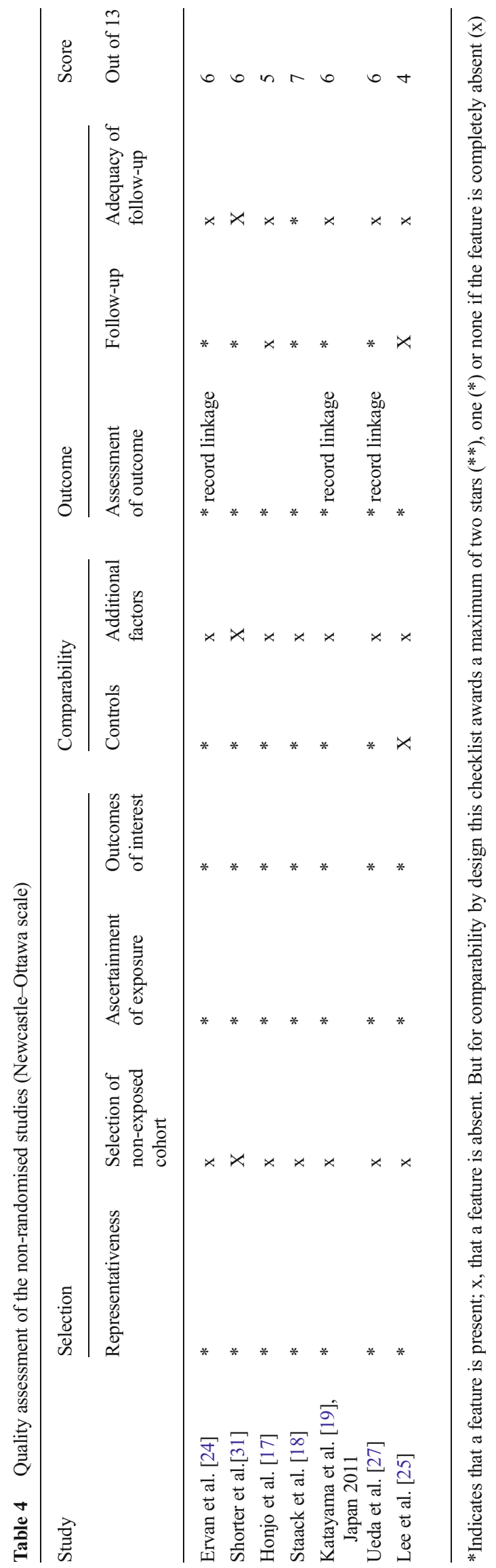

nervous system. Patients with BPS display tension and tenderness of the pelvic floor musculature and connective tissue restrictions of muscle, fascia and subcutaneous tissue of the pelvic floor. Physical therapies, such as MPT, were found to be beneficial in women with symptoms of BS and associated pelvic floor tenderness [23].

\section{Discussion}

To date there has been a paucity of studies addressing the effectiveness of complementary therapies in BPS. Results obtained from the small-scale studies we reviewed must be considered with caution because of the number of participants. Moreover, the definition of BPS in various studies is not uniform. Several unanswered questions regarding complementary therapies exist including, its applicability to all patients with BPS and the costeffectiveness of treatment. The majority of studies reviewed undertook a limited period of follow-up prohibiting analysis of the long-term efficacy of the treatment. Studies evaluating the role of tibial nerve stimulation were excluded, as this has been explored in a recent systematic review [28].

The studies included did not discuss details of adverse effects of complementary therapies. However, in 2009 a group of CAM researchers conducted a workshop to discuss the challenges and safety issues of CAM. They found a low incidence of harmful/ serious side effects from CAM [29]. Prospective observational studies have been conducted to evaluate the harmful effects of acupuncture; it has been found to be relatively safe. The data from 2.2 million treatment sessions found a risk of harmful events to be 1 in 76,000 patients [30]. The most common side effects noted with acupuncture were minor, e.g. bleeding or haematoma.

Shorter et al. utilised a validated questionnaire (IC/nutrition questionnaire) in a survey to detect whether food, beverages and supplement affect bladder symptoms in patients with BPS [31]. Analysis of the scoring of individual comestible items revealed that most bothersome consumables were caffeinated, carbonated and alcoholic beverages, citrus fruits and juices, artificial sweeteners and spicy foods. These results were obtained from survey rating and therefore further research and studies are required for confirmation of the results of the surveys conducted. Relief of symptoms was noted following ingestion of alkaline agents, e.g. calcium glycerophosphate (Prelief ${ }^{\circledR}$ ) or sodium bicarbonate (baking soda). Interestingly, $75 \%$ of respondents indicated that they had allergies, including seasonal allergies, allergies to medications, animals or foods. The survey conducted on female twins $(n=9,349)$ found that tea consumption was associated with an increased risk of BPS (OR 1.74, 95\%CI 1.24-2.44), although coffee consumption was not (OR 1.1, 95\%CI 0.84-1.45) [32]. Former and current smoking was associated with a higher risk of BPS (OR 1.5, 95\%CI 1.18-1.89 and OR 1.49, 95\%CI 1.16-1.92 respectively) [33]. 
In 2009, the Interstitial Cystitis Association (ICA) conducted an Internet-based survey of complementary therapies. A total of 2,101 responded to the survey; 1,982 confirmed an IC diagnosis. $84.2 \%$ had tried complementary therapies and $55 \%$ said that physicians had recommended complementary therapies [4]. Therapies perceived to be helpful included dietary management, myofascial physical therapy, temperaturerelated therapies, acupuncture, stress reduction and exercise. Some supplements, such as calcium glycerophosphate (Prelief), vitamin D, fish oil and probiotics, were also considered to be helpful. Calcium glycerophosphate appears to reduce BPS symptoms in a patient whose symptoms are exacerbated by particular foods [34]. Tettamanti et al. reported their findings from a population-based study demonstrating that tea and smoking were positively associated with BPS [33].

Many interstitial cystitis (IC) patients resort to nonconventional therapies after the failure of conventional measures. Unfortunately, little research on complementary and alternative therapies in the treatment of IC exists [5] and this is confirmed by our systematic review. Complementary and alternative medical therapy for interstitial cystitis (IC) is multimodal and individualized. Patients should be advised about the lack of robust evidence on complementary therapies and encouraged to participate in on-going studies on various therapies related to BPS [35].

In view of the dearth of robust evidence, there is a need for adequately powered RCTs to assess the effectiveness of complementary therapies in women with BPS. Studies could focus on therapies demonstrating potential with the limited evidence to date including: L-arginine, acupuncture, hypnotherapy and dietary modifications.

Acknowledgements Dr Seema A. Tirlapur, specialist registrar from Barts and the London School of Medicine and Dentistry, for giving us the idea and for her help with the literature search.

\section{Compliance with ethical standards}

Funding None.

\section{Conflicts of interest None.}

Open Access This article is distributed under the terms of the Creative Commons Attribution 4.0 International License (http:// creativecommons.org/licenses/by/4.0/), which permits unrestricted use, distribution, and reproduction in any medium, provided you give appropriate credit to the original author(s) and the source, provide a link to the Creative Commons license, and indicate if changes were made.

\section{References}

1. Van de Merwe JP, Nordling J, Bouchelouche P et al (2008) Diagnostic criteria, classification, and nomenclature for painful bladder syndrome/interstitial cystitis: an ESSIC proposal. Eur Urol 53(1):60-67

2. Haylen BT, de Ridder D, Freeman RM, Swift SE, Berghmans B, Lee J et al (2010) An International Urogynecological Association (IUGA)/International Continence Society (ICS) joint report on the terminology for female pelvic floor dysfunction. Neurourol Urodyn 29(1):4-20

3. The cystitis and overactive bladder foundation. Accessed 26 June 2015. Available from: http://www.cobfoundation.org/interstitialcystitispainful-bladder-syndrome

4. O'Hare PG 3rd, Hoffmann A, Allen P, Gordon B, Salin L, Whitmore K (2013) Interstitial cystitis patients' use and rating of complementary and alternative medicine therapies. Int Urogynecol J 24(6):977-982

5. Whitmore KE (2002) Complementary and alternative therapies as treatment approaches for interstitial cystitis. Rev Urol 4(Suppl 1): S28-S35

6. Ernst E, Resch KL, Mills S, Hill R, Mitchell A, Willoughby M et al (1995) Complementary medicine - a definition. Br J Gen Pract 45(398):506

7. Moher D, Liberati A, Tetzlaff J, Altman DG, Group P (2009) Preferred reporting items for systematic reviews and meta-analyses: the PRISMA statement. BMJ 339:b2535

8. Williamson I (2008) "EndNote", software for small business. Windows and Vista programs to help you improve efficiency and productivity. Productive Publications. Accessed 14 May 2012

9. Higgins JP, Altman DG, Gotzsche PC, Juni P, Moher D, Oxman $\mathrm{AD}$ et al (2011) The Cochrane Collaboration's tool for assessing risk of bias in randomised trials. BMJ 343:d5928

10. Deeks JJ, Dinnes J, D'Amico R, Sowden AJ, Sakarovitch C, Song F et al (2003) Evaluating non-randomised intervention studies. Health Technol Assess 7(27):iii-x, 1-173

11. Wewers ME, Lowe NK (1990) A critical review of visual analogue scales in the measurement of clinical phenomena. Res Nurs Health 13(4):227-236

12. O'Leary MP, Sant GR, Fowler FJ Jr, Whitmore KE, Spolarich-Kroll $\mathrm{J}(1997)$ The interstitial cystitis symptom index and problem index. Urology 49 [5A Suppl]:58-63

13. Hanash KA, Pool TL (1969) Interstitial cystitis in men. J Urol 102(4):427-428

14. Hunner GL (1918) A rare type of bladder ulcer: further notes and a report of 18 cases. JAMA J Am Med Assoc 70:208-212

15. Messing EM, Stamey TA (1978) Interstitial cystitis: early diagnosis, pathology, and treatment. Urology 12(4):381-392

16. Wein AJ, Hanno P, Gillenwater JY (1990) Interstitial cystitis: an introduction to the problem. In: Hanno PM, Staskin DR, Krane RJ, Wein AJ (eds) Interstitial cystitis. Springer, London, pp 3-15

17. Honjo H UO, Kitakoji H, Ushijima S, Kawauchi A, Nakao M, Miki $T$ (2007) Sacral acupuncture normalized bladder sensory dysfunction of $\mathrm{C}$-fiber in patients with overactive bladder and painful bladder syndrome. http://www.ics.org/Abstracts/Publish/45/000408.pdf

18. Staack A, Twiss C, Arboleda V et al (2010) Acupuncture for the treatment of interstitial cystitis - a pilot study. Female Pelvic Med Reconstr Surg 16(5):S163

19. Katayama Y, Nakahara K, Shitamura T, Mukai S, Wakeda H, Yamashita Y et al (2013) Effectiveness of acupuncture and moxibustion therapy for the treatment of refractory interstitial cystitis. Hinyokika Kiyo Acta Urol Jpn 59(5):265-269

20. Doggweiler R (2006) Hypnotherapy as a complementary treatment of interstitial cystitis/chronic pelvic pain syndrome. International Continence Society http://www.ics.org/Abstracts/Publish/44/ 000237.pdf

21. Carrico DJ, Peters KM, Diokno AC (2008) Guided imagery for women with interstitial cystitis: results of a prospective, randomized controlled pilot study. J Altern Complement Med 14(1):53-60 
22. Matsumoto S, Ueda T, Kakizaki H (2013) Effect of supplementation with hydrogen-rich water in patients with interstitial cystitis/ painful bladder syndrome. Urology 81(2):226-230

23. FitzGerald MP, Payne CK, Lukacz ES, Yang CC, Peters KM, Chai $\mathrm{TC}$ et al (2012) Randomized multicenter clinical trial of myofascial physical therapy in women with interstitial cystitis/painful bladder syndrome and pelvic floor tenderness. J Urol 187(6):2113-2118

24. Ervan K, Arcilla G, Fortman C, Dillavou H, Fortman C, Kotarinos E, FitzGerald MP, Kotarinos R (2006) Physical therapy can reduce symptoms of painful bladder syndrome. Loyola University Medical Center, Chicago

25. Lee MH, Chen WC, Lee SP, Chen YC, Liu WB et al (2012) Combination of pelvic floor biofeedback with electric stimulation can improve chronic pelvic pain, lower urinary tract symptoms and sexual dysfunction in patients with interstitial cystitis/bladder pain syndrome (IC/BPS) - 1 year follow up. International Continence Society http://www.ics.org/Abstracts/Publish/134/000731.pdf

26. Korting GE, Smith SD, Wheeler MA, Weiss RM, Foster HE Jr (1999) A randomized double-blind trial of oral L-arginine for treatment of interstitial cystitis. J Urol 161(2):558-565

27. Ueda T, Yoshida T, Tanoue H, Ito M, Tamaki M, Ito Y et al (2014) Urine alkalization improves the problems of pain and sleep in hypersensitive bladder syndrome. Int J Urol 21(5):512-517

28. Tirlapur SA, Vlismas A, Ball E, Khan KS (2013) Nerve stimulation for chronic pelvic pain and bladder pain syndrome: a systematic review. Acta Obstet Gynecol Scand 92(8):881-887
29. White A, Boon H, Alraek T, Lewith G et al (2014) Reducing the risk of complementary and alternative medicine (CAM): challenges and priorities. Eur J Intern Med 6(4):404-408

30. Witt CM, Pach D, Brinkhaus B, Wruck K, Tag B, Mank S et al (2009) Safety of acupuncture: results of a prospective observational study with 229,230 patients and introduction of a medical information and consent form. Forsch Komplementmed 16(2):91-97

31. Shorter B, Lesser M, Moldwin RM, Kushner L (2007) Effect of comestibles on symptoms of interstitial cystitis. J Urol 178(1):145152

32. Lichtenstein P, Sullivan PF, Cnattingius S, Gatz M, Johansson S, Carlstrom E et al (2006) The Swedish Twin Registry in the third millennium: an update. Twin Res Hum Genet 9(6):875-882

33. Tettamanti G, Nyman-Iliadou A, Pedersen NL, Bellocco R, Milsom I, Altman D (2011) Influence of smoking, coffee, and tea consumption on bladder pain syndrome in female twins. Urology 77(6): 1313-1317

34. Bologna RA, Gomelsky A, Lukban JC, Tu LM, Holzberg AS, Whitmore KE (2001) The efficacy of calcium glycerophosphate in the prevention of food-related flares in interstitial cystitis. Urology 57 [6 Suppl 1]:119-120

35. Nickel JC, Jain P, Shore N, Anderson J, Giesing D, Lee H et al (2012) Continuous intravesical lidocaine treatment for interstitial cystitis/bladder pain syndrome: safety and efficacy of a new drug delivery device. Sci Transl Med 4(143):143ra00 